\title{
Optimization Design of UAV Flight Control System Based on Baseline Drift Suppression
}

\author{
Lu Xing-Hua ${ }^{1,}$, Zheng Chang-hong ${ }^{2, b}$, \\ ${ }^{1}$ Huali College Guangdong University of Technology, Guangzhou Zengcheng, 511325, P. R. China \\ a44680189@qq.com, b549997208@qq.com
}

Keywords: unmanned aerial vehicle,control system,baseline drift,system design.

\begin{abstract}
In the unmanned aerial vehicle control system design, the controller design is the base of improving the control stability performance, considering the nonlinear and uncertain properties of the model, the beam space weighted spectral peak searching method is used to estimate the unknown parameters of the object. In the control system, when the pulse frequency becomes higher, signal DC component coupling capacitor will drift to the zero level, which produces baseline drift. This paper presents an UAV flight control system design optimization method based on baseline drift suppression. Using a self buffer DC baseline restorer to reduce the time constant of the discharge circuit, accelerates the discharge process, improves the control performance, and prevents baseline drift. The overall design description of UAV flight control system is analyzed, function and parameters are presented, and the hardware module of control system is designed. The performance of the system is tested by simulation. The simulation results show that, the control system can effectively inhibit the baseline drift of UAV flight control system, it has high control precision, the UAV pitch angle, and rudder angle have better tracking performance.
\end{abstract}

\section{Introduction}

With the development of aircraft design and manufacturing technology, machine production and design techniques have get comprehensive promotion, now unmanned aerial vehicle (UAV) has been widely used in the field of aerospace, target recognition, intelligent detection and field investigation, due to the characteristics of the UAV good mobility, small size, light weight, and hidden strong, etc., in the implementation of the risky task, it shows good application value. In the field of military reconnaissance, hazard detection, it is widely used, and showing a good application prospects. Unmanned aerial vehicle is the product of highly developed modern industry and informatization, the UAV is realized by artificial intelligence automatic control, operation of corresponding difficulty instead of human being. Now, unmanned aerial vehicle has good application value in field of polar expedition, space exploration and underwater detection.

UAV control system design is the core of UAV design, the operating environment of UAV is relatively complex, and it is needed to design effective UAV control method to improve the control precision of UAV behavior. Control and flight path planning for unmanned aerial vehicle is motion planning problem with multiple constraints. The traditional control method used sensors by sensitive element to realize drive and execution system linkage control, but the control system prone to nonlinear distortion, resulting in baseline drift of UAV control. In this regard, the relevant references have made the improvement on the UAV control system. Among them, the reference [3] proposed UAV flight guidance trajectory tracking control method based on neural network reinforcement learning, the DSP chip is used to design the circuit, but the control system of UAV longitudinal path planning and autonomous positioning performance is not good. In reference [4], it designed an UAV control execution system with acoustic electric conversion and attitude control, mainly including the array, duplexer and a power amplifier part, by controlling the signal will switch the signal transmitter realize man-machine nonlinear control, to improve the stability of the system, but the control system had heavy interference in the analog signal pretreatment. UAV flight control system, which is under the strong disturbance of air current, puts forward a more stringent requirements on the search of 
UAV, which leads to poor control performance. In the hardware design, it needs for disturbance suppression.

In this paper, the hardware system design method of UAV flight control system is studied, and a method of UAV flight control system is proposed based on baseline drift suppression. Using a self buffer DC baseline restorer, by reducing the time constant of the discharge circuit, accelerate the discharge process, improve the control performance, and prevent baseline drift. Firstly, the overall design description and function analysis of the UAV flight control system are carried out, and the hardware modules of the control system are designed. Finally, simulation tests are carried out to demonstrate the superior performance of the proposed method.

\section{Overall design of the system and description of device selection}

\subsection{General design description of hardware design of UAV control system}

This chapter mainly discusses the hardware design of UAV control system, according to the working characteristics of UAV control system, select the appropriate digital signal processor and peripheral circuit devices, on the basis of this, using the method of modular design of system hardware, including: DSP minimum system design, module design, A/D module baseline restoration design, D/A module design, CAN module design and system design of power module. In the military and aerospace applications for high performance and high reliability, minimize the use of high performance DSP processor in UAV control system design, ADSP-BF537 from ADI company is selected. Blackfin series DSP which belongs to ADI company, which is the main latest product recently pushed, micro signal architecture jointly is developed by ADI and Intel company based on MSA, it will be a 32 bit RISC instruction set and dual 16 bit multiplier accumulator (MAC) together, it fully integrated the signal processing function and the general type micro controller function.

ADSP-BF537BBC-5A is an industrial grade 16 DSP. According to the selection of the core components, the overall design of the system is described, the UAV longitudinal nonlinear control system is mainly composed of sensing system, driver and actuator system, and control center unit subsystems. The perception system is composed the original devices for human-computer access to sense the outside information longitudinal with nonlinear control system, it is the sensor, to achieve useful information for input information from the outside world through perception, it provides the central nervous system that the UAV longitudinal nonlinear control system control center for processing unit. It can provide guidance for the implementation of control execution unit. Detection and the input of signal can be obtained by sensor and transducer, drive and execution system is the output mechanism of UAV longitudinal nonlinear control system, which is controlled by the gyro, relay, solenoid valves, and heart unit control of UAV longitudinal nonlinear control system is the central nervous system, which is equivalent to the human brain. It sends the control command to the control system, which makes the whole UAV control system works according to the established plan.The central control unit is composed with DSP, ARM single chip processor, as described above, the overall structure of the UAV longitudinal nonlinear control system is shown in Figure 1.

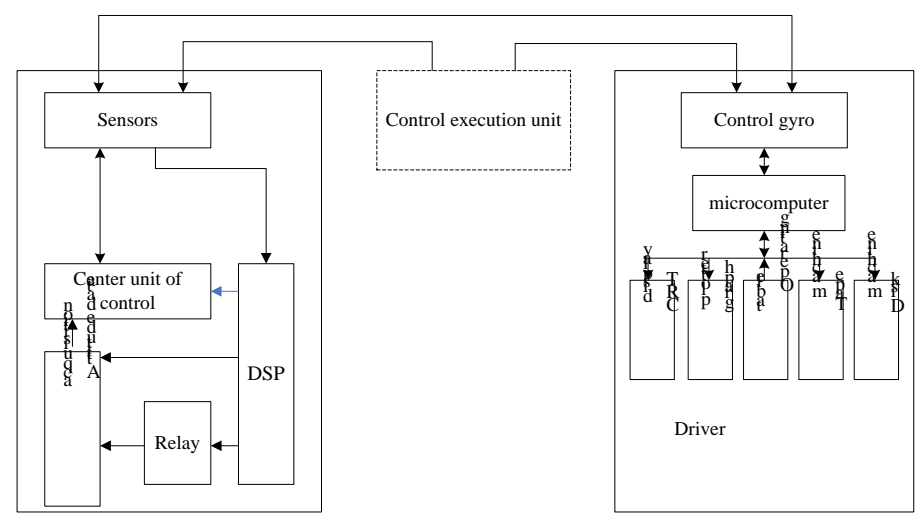

Figure 1. Overall structure of UAV control system 
On the basis of the description of the overall structure, the research of UAV longitudinal nonlinear control principle is taken, considering the nonlinear characteristics of UAV control system and uncertainty in the tracking control, the UAV generally consists of the information fusion model for $n$ decision variables, the input and output model of the system can fuse longitudinal nonlinear control parameters, the input parameters are $u(n), w_{0}^{*} \ldots w_{M}^{*}$, the output variable is $y(n)$, it is the quantitative characteristics of UAV attitude.

\subsection{Analysis of device selection and function of UAV control system}

In the control system, when the pulse frequency becomes higher, signal DC component coupling capacitor will drift to the zero level, which produces baseline drift. This paper presents an UAV flight control system design optimization method based on baseline drift suppression. Using a self buffer DC baseline restorer to reduce the time constant of the discharge circuit, accelerates the discharge process, improves the control performance, and prevents baseline drift. By using the method of modular system hardware design, including: DSP information processing system design, A/D module design, D/A module design, CAN communication module and system power module design, control system is a broadband system, including sending and receiving circuit, analog signal pre processor, data acquisition system, power amplifier etc.. The controller adopts the embedded controller PXI-8155 controller provides a rich and extensible interface such as serial port, parallel port, USB port, mouse, keyboard port, Ethernet interface and GPIB interface etc.. PXI-6713 is a 8 channels analog output module, a total number is 3 blocks, 24 channel data playback system is completed. The maximum output range is $-10 \mathrm{~V} \sim 10 \mathrm{~V}$, the highest update rate of $1 \mathrm{MSa} / \mathrm{s}$ playback technology, design indexes of the system are described as:

(1) The dynamic range of control signal pre processor: $-40 \mathrm{~dB} \sim+40 \mathrm{~dB}$, enlarge capacity $80 \mathrm{~dB}$, the amplitude of the output signal $\mathrm{V}$;

(2) Flight attitude data sampling channel: 8 channel synchronous and asynchronous input;

(3) Data sampling rate: $\geq 200 \mathrm{KHz}$;

(4) A/D resolution: 12 (at least);

(5) D/A resolution: 12 (at least);

(6) $\mathrm{D} /$ A conversion rate: $>200 \mathrm{KHz}$;

(7) Control signal playback options, including: (CW, LFM, HFM) in various forms.

Based on the above requirements, peripheral devices this system chose ADI's high-speed A/D chip AD9225, the sampling frequency is $25 \mathrm{MHz}, 12$ bit resolution, using a single $5 \mathrm{~V}$ power supply, the input range is $4 \mathrm{Vpp}$, the power consumption is $280 \mathrm{~mW}$, fully meet the requirements.

\section{Design and implementation of the hardware circuit of the system}

According to the above design idea, put forward the design scheme of this new type of UAV flight control system, due to the exchange coupling, is it is inevitable to produce baseline drift, the baseline drift correction baseline restoration circuit is designed. In order to increase the drive capability, adding a follower before the A/D converter. High speed A/D converter makes high speed sampling on the input signal, and then through the DMA way to the DSP internal buffer, it is a double buffer settings form, when filled with a buffer, DMA automatic sends a buffer fill number, while DSP peak detection is taken with a data buffer. According to the working principle of the traditional UAV control system, the sampling method is peak sampling, only sampling the peak voltage of the pulse signal, the peak judgment is simulated by discrete circuits, which has high power consumption and temperature drift etc.. This design abandons the traditional practice, the high speed signal processor and high speed A/D are used, the original signal from the scintillation probe output directly to complete high-speed sampling, and then through the software method, peak detection is obtained, so as to improve the system stability and anti-jamming, avoid drift phenomenon, and the system is easy to upgrade. The design structure of the digital acquisition amplifier in the control system is shown in figure 2 . 


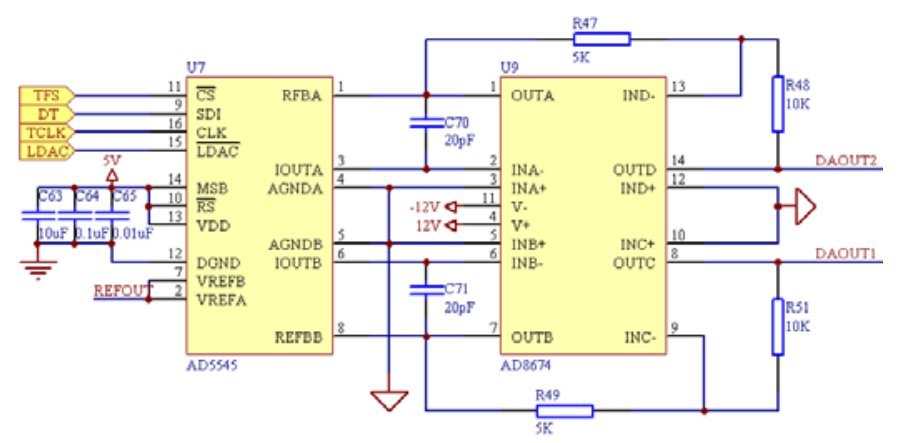

Figure 2. Design structure of the digital acquisition amplifier in the control system

Select B380C EM as the interface chip, in the nonlinear control system of the interference filter part, using MAX264 filter, there are two independent two order filter, it can be cascaded to achieve high order filter. The design circuit of the filter is shown in figure 3. In Figure 3, we can use the chip clock in the external crystal, the input clock frequency as two internal frequencies. On M0,M1 two pin programming can make the chip work in pattern 1, 2, 3, 4 several ways, after DC-DC conversion to $5 \mathrm{~V}, 5 \mathrm{~V}$ DC after DC-DC get $3.3 \mathrm{~V}$ and voltage $4.2 \mathrm{~V}$, the control system of self correction model reference adaptive control filter is obtained.

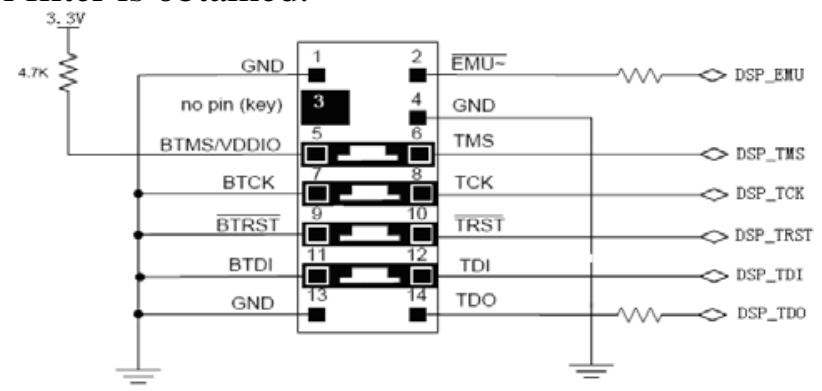

Figure 3. Self tuning model reference adaptive control filter

Based on this, the design error compensator is designed to control the steady state error of the UAV longitudinal nonlinear control system in a small range. Through the above analysis, the optimization design of the control system is realized.

\section{Simulation test and performance analysis}

In order to test the performance of the UAV control system designed in this paper, the simulation experiment is carried out. First, the DSP IO port is configured, set LDAC signal to a low level to ensure write D/A data switch immediately, then the DSP through the serial port to send ad5545 a 0x3FFFF, set two D/A and output maximum value as $5 \mathrm{~V}$, wait for 200000 NOP instructions, again to send a $0 \times 30000$ ad5545 arranged two D/A output at the same time, the minimum value is $0 \mathrm{~V}$, wait for 200000 NOP instructions and then transmits the maximum value, with the cycle, thus forming continuous square wave, pitch angle, rudder angle and other parameters tracking performance curve of the UAV control system is shown in Figure 4. It shows that the control system can effectively inhibit the baseline drift of the UAV flight control, the control precision is high, the UAV pitch angle, rudder angle and other parameters have good tracking performance. Simulation results demonstrate the superior performance of the design system in this paper.

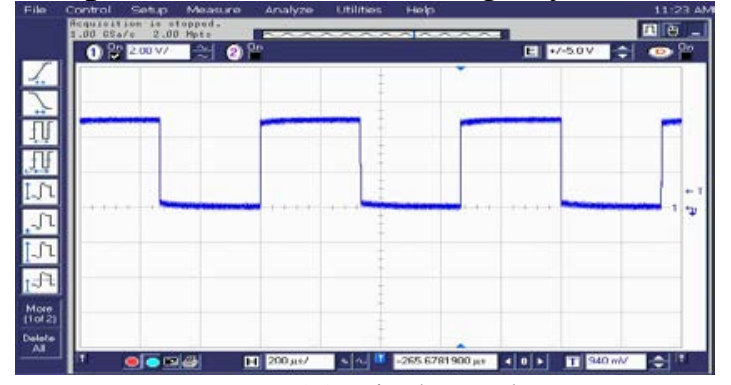

(a) Pitch angle

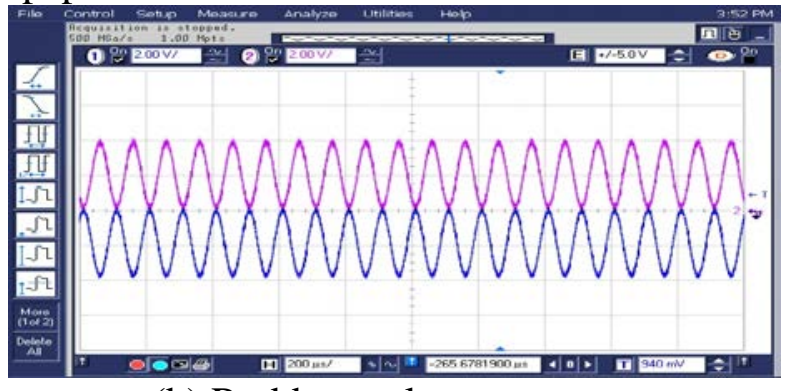

(b) Rudder angle

Figure 4.Parameter tracking test curve for UAV Control 


\section{Conclusions}

An UAV flight control system design optimization method is proposed based on baseline drift suppression. Using a self buffer DC baseline restorer to reduce the time constant of the discharge circuit, accelerates the discharge process, improves the control performance, and prevents baseline drift. The overall design description of UAV flight control system is analyzed, function and parameters are presented, and the hardware module of control system is designed. The performance of the system is tested by simulation. The simulation results show that, the control system can effectively inhibit the baseline drift of UAV flight control system, it has high control precision, the UAV pitch angle, and rudder angle have better tracking performance.

\section{Acknowledgments}

This project is supported by Foundation for Distinguished Young Talents (Natural Science, 2015KQNCX218)of 2015 Key Platforms and Research Projects of Department of Education of Guangdong Province and 2016 Undergraduate Scientific and Technological Innovation Project Fund of Guangdong Province (pdjh2016b0933). 2012 Guangdong Quality Projects the Electrical and Mechanical Services Skills Training Center (Cantonese teaching high honor [2012]204)

\section{References}

[1] ROGER W PRATT.Flight control systems practicalissues in design and implementation[M]. United Kingdom.The Institution of Electrical Engineers, 2000: 44-49.

[2] Pradeep B, Naomi E L. Nonlinear gliding stability and control for vehicles with hydrodynamic forcing[J]. Automatica, 2008, 44: 1240-1250.

[3] Matheny M H, Grau M, Villanueva L G, et al. Phase synchronization of two anharmonic nanomechanical oscillators[J]. Physical Review Letters, 2014, 112(1): 014101.

[4] Engel J, Sturm J, Cremers D. Camera-based navigation of a low-cost quadrocopter[C]//2012 IEEE/RSJ International Conference on Intelligent Robots and Systems. Piscataway, NJ, USA: IEEE, 2012: 2815-2821.

[5] Weiss S, Achtelik M W, Lynen S, et al. Monocular vision for long-term micro aerial vehicle state estimation: A compendium[J]. Journal of Field Robotics, 2013, 30(5): 803-830.

[6] Ghadiok V, Goldin J, Ren W. On the design and development of attitude stabilization, vision-based navigation, and aerial gripping for a low-cost quadrotor[J]. Autonomous Rob s, 2012, 30(1/2): 41-68. 